\title{
Pulmonary sequestration at the posterior mediastinum in a neonate
}

\author{
Lu-Ting Kuo, MD, PhD, ${ }^{a}$ Chien-Hwa Chang, MD, ${ }^{a}$ Kuan-Ting Kuo, MD, ${ }^{b}$
}

Dung-Cheng Chang, MD, and Hong-Shiee Lai, MD, PhD, ${ }^{a}$ Taipei, Taiwan

$\mathrm{P}$ ulmonary sequestration (PS) is a condition that may remain asymptomatic and never be diagnosed. Extralobar sequestration (ELS) accounts for $25 \%$ of all sequestrations and may present with respiratory distress, feeding difficulties, or congestive heart failure in the first 6 months of life. It is usually identified by routine sonography when screening for other congenital anomalies and may occur in the thoracic cavity or above or below the diaphragm. ${ }^{2}$ However, it is rarely reported in the mediastinum, especially at the neonatal stage. We now describe an asymptomatic newborn with ELS in the posterior mediastinum.

From the Departments of Surgery ${ }^{\mathrm{a}}$ and Pathology, ${ }^{\mathrm{b}}$ National Taiwan University Hospital and National Taiwan University College of Medicine, Taipei, Taiwan.

Received for publication Dec 6, 2005; accepted for publication Jan 13, 2006.

Address for reprints: Hong-Shiee Lai, MD, PhD, Department of Surgery, National Taiwan University Hospital, No. 7, Chung-Shan South Road, Taipei, Taiwan (E-mail: hslai@ha.mc.ntu.edu.tw).

J Thorac Cardiovasc Surg 2006;132:185-7

$0022-5223 / \$ 32.00$

Copyright (C) 2006 by The American Association for Thoracic Surgery doi:10.1016/j.jtcvs.2006.01.058

\section{Clinical Summary}

A male fetus was diagnosed with a hyperechogenic mass in the retroperitoneal or mediastinal space by antenatal ultrasonography during routine prenatal examinations at 28 weeks of gestational age in a gravida 2, para 2 woman (Figure 1, A). The mother had no family history of congenital abnormalities, and her pregnancy was uneventful. The biophysical profile of the fetus was normal, and he was born by spontaneous vaginal delivery at 37 weeks of gestation. His birth weight was $2950 \mathrm{~g}$, and his APGAR score was 9 at 1 minute and 10 at 5 minutes after delivery. No other congenital anomalies were noted on postnatal physical examination, which disclosed no abnormality.

Magnetic resonance imaging demonstrated a hyperintense mass compared with the normal lung parenchyma and located in the paravertebral region, attached to the descending aorta in the posterior mediastinum (Figure 1, B, C). However, magnetic resonance angiography and color Doppler ultrasound failed to detect the origin of the supplying or draining vessels. Differential diagnosis included ELS, bronchogenic cysts, congenital cystic adenomatoid malformation, enteric duplication, neuroblastoma, hemangioma, lymphangioma, and teratoma. The infant underwent surgery at the age of 40 days.
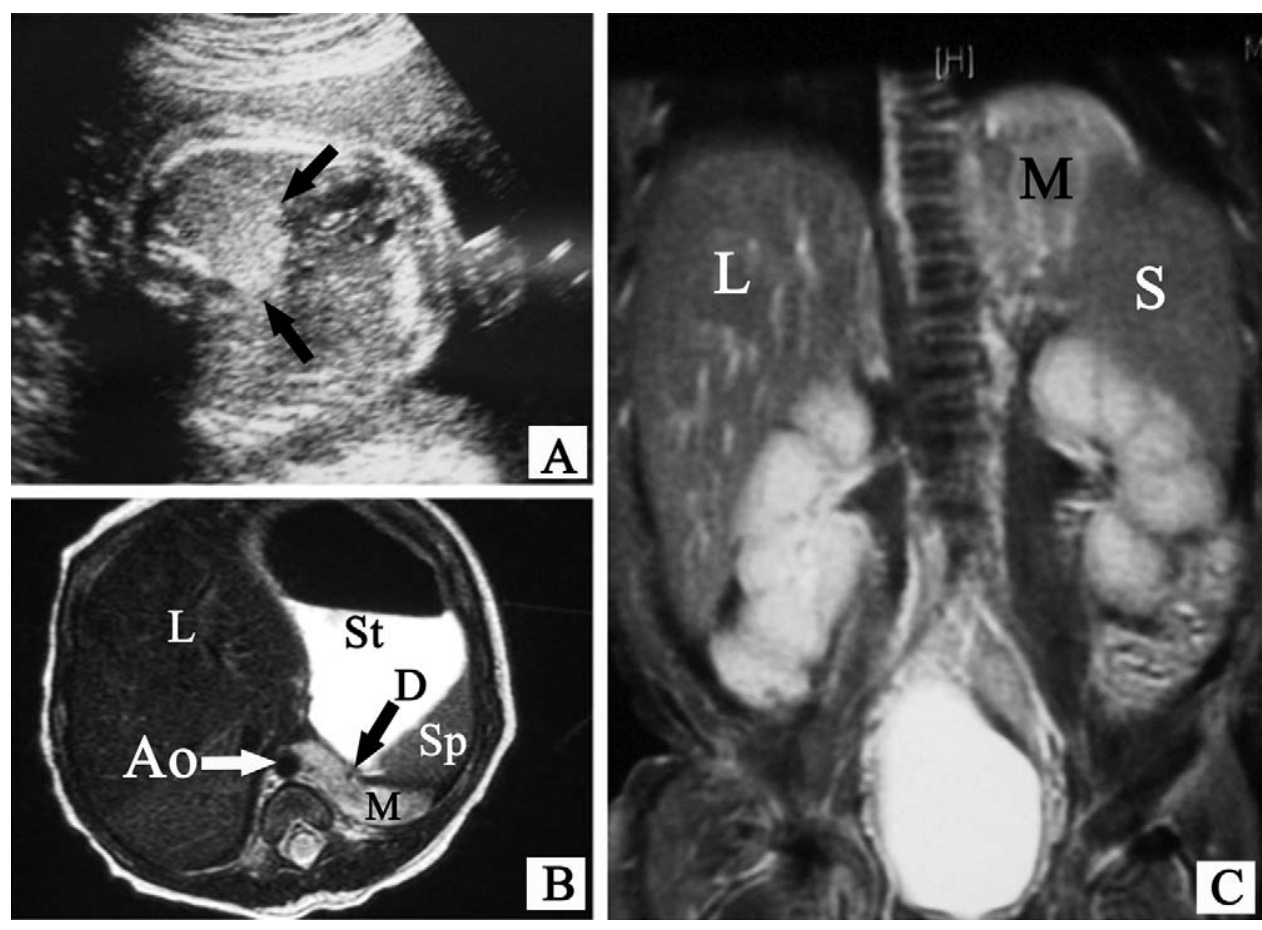

Figure 1. A, Prenatal ultrasound of the fetus at gestational week 28 showed an echogenic mass (arrows) in the retroperitoneal or mediastinal space. Axial (B) and coronal (C) magnetic resonance imaging showed a well-defined mass, moderately enhanced by gadolinium, located in the left paravertebral region and attached to the descending aorta in the posterior mediastinum. Ao, Aorta; $D$, diaphragm; $L$, liver; $M$, mass; $S t$, stomach; $S p$, spleen. 

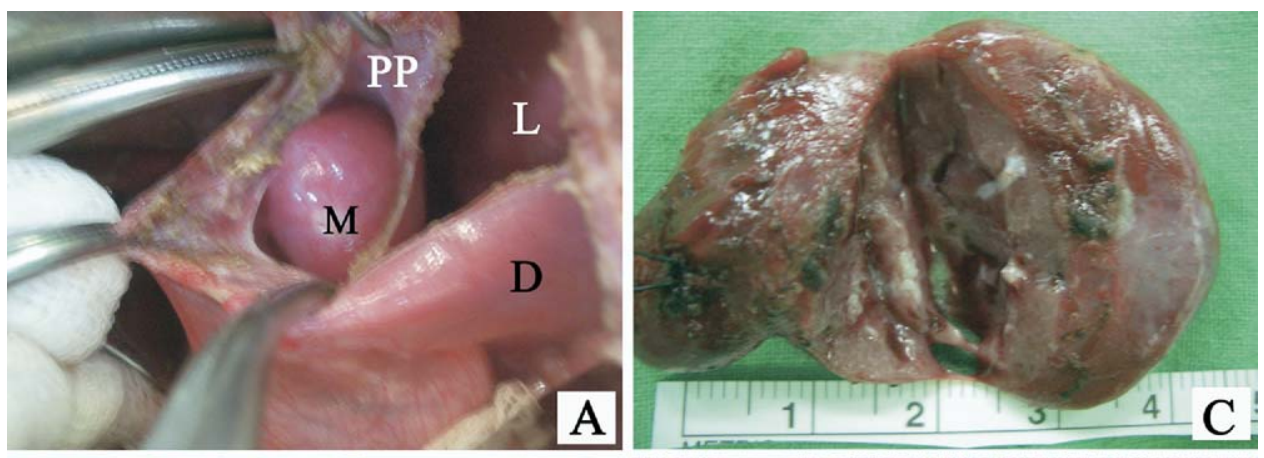

Figure 2. A, Mass at the posterior lower mediastinum. B, Small feeding artery (arrow) arising from the descending aorta $\left(^{*}\right)$ to supply the mass. C, Cut surface of the resected mass shows a collapsed lung. D, Histologic picture shows a bronchial structure $(*)$ with an island of cartilage (arrow) in the wall, dilated Iymphatic channels (\#), and an artery (§). D, Diaphragm; $L$, lung; $M$, mass; $P P$,
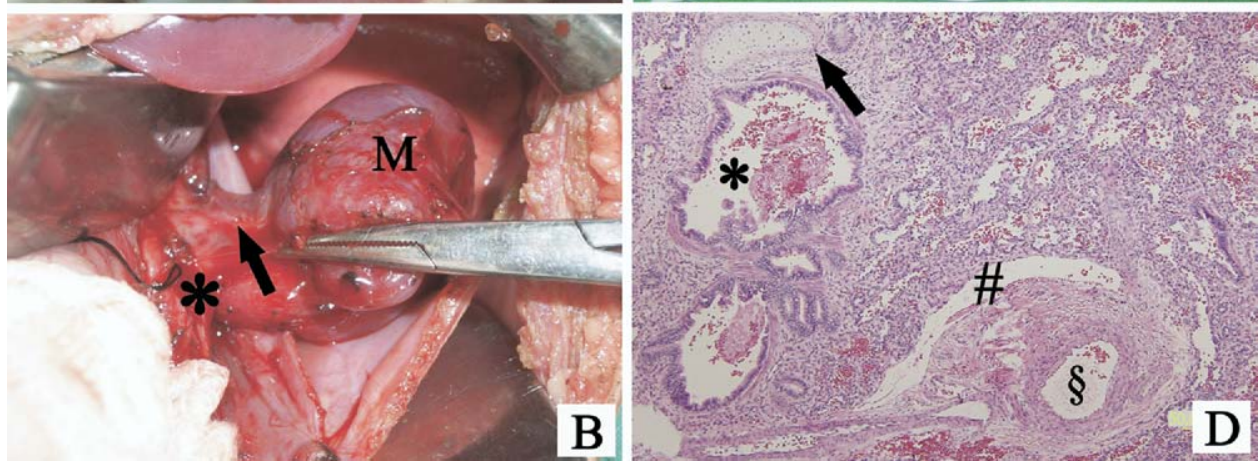
parietal pleura.

Through a transverse laparotomy, no diaphragmatic hernia or mass lesion was seen in the peritoneal cavity. The left hemidiaphragm and the mediastinal surface of the medial parietal pleura were then opened. A pyramid-shaped, smooth, soft, well-encapsulated mass, measuring $2 \times 3 \times 3 \mathrm{~cm}$, was found attached to the descending aorta and superiorly to the diaphragm (Figure 2, $A, B$ ). One short feeding artery originating from the aorta was identified and then ligated and divided before excision of the mass. The cut surface of the mass was spongy-like (Figure 2, C).

Microscopically, a malformed bronchial structure containing mucin with cartilage in its wall, with uniformly dilated bronchioles, alveolar ducts, and alveoli, were demonstrated (Figure $2, D)$. There was a high ratio of mesenchyma to air space; this was diagnosed as a sequestrated pulmonary lobe. The postoperative course was uneventful, and the child is currently well 8 months after the surgery.

\section{Discussion}

Approximately $90 \%$ of ELS has been reported to be in the left thorax, between the lower lobe of the lung and the diaphragm, especially involving the posterior costophrenic sulcus. ${ }^{1}$ Associated congenital abnormalities are common in more than $60 \%$ of patients with ELS. Only $8 \%$ of extralobar sequestrations are intra-abdominal or retroperitoneal, which are sometimes associated with diaphragmatic hernia and are diagnosed in the first decade of life. With routine prenatal ultrasonographic examination, ELS could possibly be detected prenatally with increasing frequency.

Only 3 cases of extralobar PS in the posterior mediastinum have been reported, ${ }^{3-5}$ but none of these were at the lower posterior mediastinum. These patients included 2 women, aged
45 and 48 years, and a man aged 65 years, with ELS bounded anteriorly by the pericardium and posteriorly by the bodies of the thoracic vertebrae. In contrast, our patient was identified at the neonatal stage with ELS at the lower posterior mediastinum.

The definitive diagnosis was not reached before surgery in this patient. A preoperative diagnosis of PS and its supplying artery, especially the identification of the systemic artery, is important in preventing injury to the blood supply of the normal lung. Although angiography is somewhat risky for infants and young children, it might be required in patients in whom there is a failure to demonstrate the anomalous vessel by magnetic resonance angiography because of the small size of the artery or an unfavorable orientation in relation to the scanning plane. The unusual location and failure of angiographic image studies makes the preoperative diagnosis of ELS difficult. Therefore, exploratory surgery is indicated, even in an infant, to exclude other diagnoses.

\section{Conclusion}

We present a case of a neonate with ELS in an unusual location without any associated congenital abnormalities. It must be emphasized that PS is one of the differential diagnoses for asymptomatic mediastinal mass in a neonate. A thorough and comprehensive combination of imaging approaches should be used, followed by a pathologic examination of the congenital anomaly to establish a definitive diagnosis.

\section{References}

1. DeParedes CG, Pierce WS, Johnson DG. Pulmonary sequestration in infants and children: a 20-year experience and review of the literature. J Paediatr Surg. 1970;5:136-47. 
2. Chan YF, Oldfield R, Vogel S. Pulmonary sequestration presenting as a prenatally detected suprarenal lesion in a neonate. J Pediatr Surg. 2000;35:1367-9.

3. Cougard P, Bermard A, Melnick W, Guerin JC, Viard H. Cystic tumor of the mediastinum of digestive origin. Apropos of a new case. Diagnostic and pathogenic hypotheses. Ann Chir. 1992;46:774.
4. Ikeda Y, Matushita Y, Chimoto M. A case report of extralobar pulmonary sequestration associated with mediastinal bronchogenic cyst. Kyobu Geka. 1998;51:154.

5. Kamiyoshihara M, Kawashima O, Sakata S, Ishikawa S, Morishita Y. Extralobar pulmonary sequestration in the posterior mediastinum. Scand Cardiovasc. 2001;35:157-8.

\title{
Dual-layer sandwich mesh repair in the treatment of major diaphragmatic eventration in an adult
}

\author{
Angelo Di Giorgio, MD, Carlo Luigi Cardini, MD, Paolo Sammartino, MD, Simone Sibio, MD, and \\ Enzo Naticchioni, MD, Rome, Italy
}

$\mathrm{T}$ otal eventration of a hemidiaphragm is a rare anomaly in adults. This condition could be subsequent to primary or acquired phrenic nerve palsy, but often it presents as a pure degenerative muscular disease without evident signs of denervation. Surgical repair is indicated only in cases of progressive exertional dyspnea, recurrent respiratory infections, or both. Routine surgical techniques counted are plication or incision, followed by double-breast suturing performed through a low posterolateral thoracotomy or minimally invasive access. We report a case of left major eventration in a 58-year-old woman in which the vanishing of most of the diaphragmatic tissue represented an extreme condition incompatible with the performance of a standard procedure.

\section{Clinical Summary}

A 58-year-old, female heavy smoker was admitted to our department for a 6-month history of progressive exertional dyspnea and left-sided chest pain. Ten years previously, the patient had a blunt chest trauma caused by a road accident without particular complications and with complete recovery after 5 days of hospitalization. Clinical examination revealed auscultatory bruising over the lower anterolateral quadrants of the left hemithorax; other clinical signs were absent, and laboratory parameters were within normal ranges. A standard radiograph of the chest showed a high displacement of the left hemidiaphragm with contralateral mediastinal shift. A computed tomographic scan of the chest showed a major eventration of the left hemidiaphragm with compressive atelectasis of the anterior segment of the

From Dipartimento di Chirurgia "Pietro Valdoni," Università degli Studi di Roma "La Sapienza," Rome, Italy.

Received for publication Jan 18, 2006; accepted for publication Feb 13, 2006.

Address for reprints: Angelo Di Giorgio, MD, Università degli Studi di Roma "La Sapienza," Dipartimento di Chirurgia "Pietro Valdoni," Policlinico Umberto I, Via Lancisi 2-00161, Roma, Italy (E-mail: angelo. digiorgio@uniroma1.it).

J Thorac Cardiovasc Surg 2006;132:187-9

0022-5223/\$32.00

Copyright $\odot 2006$ by The American Association for Thoracic Surgery doi:10.1016/j.jtcvs.2006.02.033 lower lobe and appearance of some bilateral apical subpleural bullae. Completion computed tomographic scanning of the brain, neck, mediastinum, and whole abdomen yielded negative findings and did not show any central or peripheral nervous lesions accounting for a phrenic nerve palsy. Static and dynamic ventilatory function parameters demonstrated a mild restrictive pattern: forced expiratory volume in 1 second of 1.63 (54\%), forced vital capacity of $1.85(44 \%)$, total lung capacity of 3.08 (56\%), expiratory reserve volume of 0.42 (46\%), and functional residual capacity of 1.37 (47\%). Ventilatory scintigraphy did not reveal ventilated parenchyma in the basal segments in the lower lobe of the left lung; the right lung was totally ventilated. Bronchoscopic results were negative, and cardiac function was proved to be normal. Five days later, the patient underwent a left posterolateral thoracotomy at the seventh intercostal space with selective thracheobronchial intubation. The stomach was drained with a nasogastric tube. The intraoperative finding was a large eventration of the whole left hemidiaphragm invading about 2 thirds of the pleural cavity. The central part of the phrenic dome was reduced to a thin transparent film constituted only by pleura and peritoneum through the left colic angle, and the great omentum was visible. At first, the apex of the phrenic dome was opened through a transversal incision,

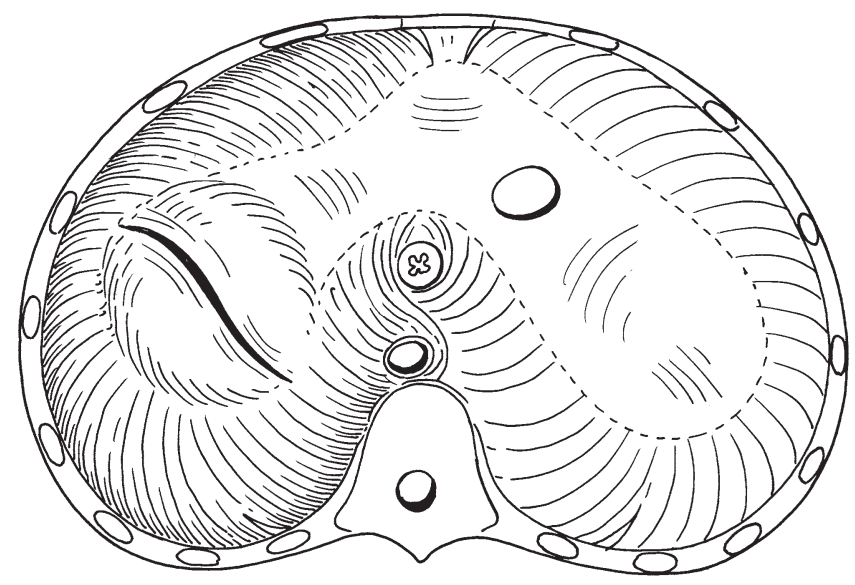

Figure 1. The apex of the phrenic dome opened through a transversal incision. 